\section{Equine colic: a review of the equine hindgut and colic}

\author{
Stephanie D. Bland \\ Department of Animal Nutrition, \\ Southern Illinois University, Carbondale, \\ IL, USA
}

\section{Abstract}

Colic is the major cause of morbidity and mortality, premature deaths, and is the number one health concern in horses. To increase the quality of life for horses, it is vital to understand the causes, signs, diagnostics, treatment, and prevention of colic. Horses are hindgut fermenters and their cecal microbiome can easily be altered due to environmental and physiological changes. Colic can be induced from starch overloading, which can have detrimental effects on the cecum microbiome, such as decreasing hindgut $\mathrm{pH}$ and altering the production of volatile fatty acids. In addition, if the immune system becomes compromised, causing changes in the hindgut microbiome, this will trigger a proliferation of opportunistic and pathogenic bacteria, which can cause numerous gastrointestinal diseases, resulting in colic. However, identifying a variety of microorganisms in the cecum is extremely difficult and understudied because difficulties in sampling and not all bacteria strains can be cultured. Therefore, next generation sequencing has helped in identifying microbes found in the gastrointestinal tract. Using the application of metagenomics, allows scientists to be able to sequence thousands of microorganisms simultaneously. In addition, exploring new techniques to help identify and achieve a clearer picture of the microbiome population and how it may change during bouts of colic could revolutionize the way veterinarians diagnose colic and tailoring a treatment specific to the microoganisms that are proliferating. Therefore, understanding the mechanisms of colic and next technological advances could increase the quality of life for horses and minimize the mortality rates.

\section{Introduction}

Colic is a gastrointestinal condition that has manifested based on a group of clinical signs from horses experiencing abdominal pain. An average of $10-11 \%$ of all horses in the United States will suffer from colic at some point in their life and has a fatality rate of $11 \% .1,2$ Treatment of colic and other related gastroin- testinal diseases is what makes the equine insurance industry a multi-million dollar market. Overloading the system with starches, bloat, addition of new supplements, and changes in the diet and the environment can all change the highly sensitive balance of the microbiome in the hindgut. This dysbiosis can cause physiological consequences to the gastrointestinal tract and the health of the animal. Knowing which factors can change the dynamics of this dense bacteria population and the signs and symptoms of colic, can provide better information to horse caretakers of what the horse is suffering from, how severe it is, and what mode of action to take. Additionally, technological advances, such as metagenomics could help in identifying specific strains of bacteria that could be causing colic. This information can ultimately improve the quality of life for horses and help decrease the rising number of cases of colic in the United States. To better understand what colic is, how it affects the horse, and measures to take for prevention; it is important to know the anatomy of a horse, how the hindgut microbes play a role in digestion, and how different factors can change those microbes.

\section{Anatomy of equine gastroin- testinal tract}

Horses are monogstric animals with a relatively small stomach. From the horse's mouth to their large intestine, their gastrointestinal tract is similar to that of a human's. However, past the cecum, a horse's gastrointestinal tract has more similarities to that of a cow's. ${ }^{3} \mathrm{~A}$ horse's gastrointestinal tract can be divided into three segments: foregut, midgut, and hindgut. 4 The foregut consists of the esophagus and stomach. Once food has passed through the stomach, it enters the small intestine (midgut): duodenum, jejunum, and ileum, which join the hindgut, cecum, colon, and rectum, at the ileocecal junction. The small intestine and stomach can almost receive a continuous flow of food, which is vital since horses are continuous grazers. ${ }^{3}$ The cecum is a large fermentation vat located on the right side of the animal. Carbohydrates, fermented by fibrolytic bacteria, produce volatile fatty acids, which account for $60-70 \%$ of the horse's total energy. However, in modern management practices, horse owners and horse caretakers do not let horses graze like they naturally should. Therefore, caretakers substitute the horse's diet with grains and fats, which the horse is not designed to properly digest. This unbalanced feeding regimen causes numerous digestive disturbances. ${ }^{3}$

Horses are classified as hindgut fermenters, meaning a balance of good and bad bacteria
Correspondence: Stephanie D. Bland, Department of Animal Nutrition, Southern Illinois University, 1205 Lincoln Drive, Carbondale, IL, 62901, USA.

Tel.: +1.618.550.9490.

E-mail: sbland@siu.edu

Key words: Colic; Horse; Metagenomics; Microbiome.

Acknowledgements: SDB would like to acknowledge Dr. Lightfoot and his AGSC 550 class for their support, encouragement, and revisions on this review.

Conflict of interest: the author declares no potential conflict of interest.

Received for publication: 19 September 2015. Revision received: 27 October 2015.

Accepted for publication: 24 November 2015.

This work is licensed under a Creative Commons Attribution NonCommercial 4.0 License (CC BYNC 4.0).

(C) Copyright S.D. Bland, 2016

Licensee PAGEPress srl, Italy

Veterinary Science Development 2016; 6:6223

doi:10.4081/vsd.2016.6223

aid in the digestion of foodstuff in the cecum and large intestine. 5 The hindgut is not only a fermentation vat, but it also stimulates the immune response, protects against pathogens, production and neutralization of toxins, and gene expression in the host's epithelial tissues. ${ }^{6}$ The cecal microbiome is extremely sensitive and can be affected by factors such as gastrointestinal disease and dietary changes, which can lead to systemic consequences and even death. ${ }^{5}$ Therefore, normal, healthy, and balanced hindgut microbiota is vital for the overall wellbeing of the animal. By understanding the external factors and how they affect the gut microbiota, could provide more efficient treatments and prognoses of gastrointestinal diseases resulting in colic.

\section{Microbiome of the equine hindgut}

Gastrointestinal microbiota play an essential role not only in digestion, but also in colonic diseases. The gut microbiome is one of the densest, most dynamic, and complex microorganism populations located in the body. 5 This unbalanced microbiome allows opportunistic and pathogenic bacteria to proliferate and can cause numerous gastrointestinal diseases, which can result in colic and 
death. In literature, the gastrointestinal microbiome, in general, has been the most studied microbiota; however, equine hindgut microorganisms have not been extensively studied. ${ }^{4}$ Only superficial knowledge exists on the equine hindgut microbiome due to a majority of bacteria that have not yet been identified because they cannot be cultured. However, with new technological advances and next generation sequencing (NGS), scientists can now achieve great strides in identifying bacteria down to the genus and species level.

\section{Next generation sequencing}

Many veterinarians and horse caretakers can recognize the symptoms of colic. However, little information has been conducted on what is happening internally, specifically to the cecum microbiome. Past studies, have used sacrificed and cannulated horses to collect cecal fluid to analyze the population of the microbiome with DNA sequencing. DNA sequencing is used to determine the exact order of nucleotides within a DNA strand. This allows scientists to study normal genes, but also compares normal genes to disease-causing versions. However, lack of technology and sequencing techniques has hindered scientists to develop a full picture of the changes to the microbiome. Currently, NGS is of the latest technology, which allows the genomic content of interest, multiple samples, to be analyzed in a single experiment, quicker and more cost effective than the past methods used for DNA sequencing. This technology parallelizes the process, running multiple DNA templates simultaneously, resulting in millions of sequences produced.

\section{Types of next generation sequencing}

First, Illumina is a type of NGS, which uses reversible dye terminator technology, which can be implemented in metagenomics. This technology is based on DNA clusters, which requires amplification of DNA in a targeted region. NGS on MiSeq technology uses a flow cell, a sandwich of two glass-slides, surface. ${ }^{7}$ In the flow cell, there are oliogonucleotides that are complimentary to the two adaptors on the DNA strand. This technique is used to hybridize and amplify the template strand, resulting in dense clusters of double stranded DNA on the flow cell. 7 Second, sequence by synthesis (SBS) is used to sequence the DNA cluster by relying on reversible terminator chemistry. In the first cycle, primers are attached to the side and amplified with polymerases. Reversible terminator bases are added one at a time and non-incorporated nucleotides are washed away. A camera in the machine takes images of the fluorescently labeled nucleotides for identification. The delayed image acquisition allows for a large array of DNA colonies to be captured by sequential images. ${ }^{7}$ Next in the process, the terminal 3' blockers are removed, allowing the next cycle to begin. Bases are added one at a time, which reduces the error rate. ${ }^{7}$ NGS also offers the option of paired-end sequencing. Paired-end sequencing, sequences DNA from both the 5' and 3' ends. The paired-end turnaround is removed at the 3' end and the lawn primer is unblocked allowing the strand to flip over, creating a bridge. The bridges are linearized and the original forward strand is cleaved and washed away, leaving the reverse strand template attached. The 3' is blocked to avoid unwanted priming. Then hybridization takes place and the cluster is ready to be sequenced in the other direction. The cycle continues in a similar manner as SBS.7

\section{Metagenomics}

NGS has helped the application of metagenomics by being able to sequence thousands of organisms simultaneously. Metagenomics, one of the fastest growing disciplines, is the study of genomic DNA and diversity from microorganism that cannot be cultured in a laboratory setting. ${ }^{8}$ Metagenomics, the process of identifying species present in a body of fluid, is used in microbiome research when collecting from the cecum. By using metagenomics, scientists are able to assemble a genome (abundance and identify) from individual microbes within the horse's cecum. This is useful during bouts of colic to be able to understand and identify the population dynamics in the cecum microbiome.

\section{Methods of metagenomics}

Metagenomics has two methods of sequencing: amplicon and shotgun sequencing. First, amplicon sequencing is widely used in studying phylogeny and taxonomy. Amplicon sequencing uses $16 \mathrm{~s}$ rRNA, which occurs in all living organisms. This particular rRNA is present in $80 \%$ of bacterial RNA and uses variable regions, which is compatible with PCR amplification and sequencing and lowers the cost. ${ }^{8}$ Probes are used to hybridize small, selected regions of the genome, typically spanning hundreds of base pairs. The downfall about amplicon sequencing is the lack of threshold identity. While some organisms may show a high sequence identity, they may have a small similarity at the genomic level. ${ }^{8}$ Secondly, is shot- gun sequencing, also called metagenome sequencing. In shotgun sequencing, DNA strands have been extracted and shred into tiny fragments before the sequencing process can begin. Shotgun sequencing uses chain termination to obtain reads. The reads become overlapped and a machine connects the overlapping reads to create a sequence. Shotgun sequencing advantages include, giving complete genomes of all the organisms in a population, accurately identifying phylogenetic information, and providing information about genes present in the population without assembling individual genomes. ${ }^{8}$ With these new technological advances, this could revolutionize how veterinarians diagnose and treat colic causes. This is vital information because it could help prevent recurrence cases and decrease mortality rates.

\section{Colic in horses}

Colic in its truest definition means abdominal pain. Overtime, the term has evolved to describe a condition rather than clinical signs. Colic includes all forms of gastrointestinal diseases that cause pain. Colic is the major cause of morbidity and mortality in horses. According to a survey in 1986, from the Morris Animal Foundation, colic is the number one health concern and a leading cause of premature deaths in horses. Annually, 10-11\% of horses suffer from colic, and $11 \%$ of cases are fatal.1,2 In 1983 , the horse insurance industry paid over $\$ 16$ million in claims, directly related to gastrointestinal problems in horses. In 2001, the cost rose to over $\$ 115,300,000$ and keeps rising. ${ }^{9}$ Therefore, to increase the quality of life for horses, it is vital to understand the causes, signs, diagnostics, treatment, and prevention of colic in horses. ${ }^{2}$

\section{Causes}

There are numerous reasons a horse can colic, including a high grain, low forage diet, moldy feed, abrupt feeding changes, internal parasite infections (strongyles and ascarids), lack of water, long term nonsteroidal antiinflammatory drugs use, stress, dental problems, and long term antibiotic use, which can affect the hindgut microbial population. ${ }^{2}$ Horses can also colic from torsion, twisting of the intestines, stomach ulcers, and uterine pain. Theses changes to a horse can cause one of four conditions, which result in abdominal pain: i) the wall of the intestine is overly stretched either by gas, fluid, or ingesta. Stretch sensitive nerve endings located within the intestinal wall will transmit pain to the 
brain; ii) presence of intestinal displacement causing extensive distortion and tension on the mesentery walls, resulting in pain; iii) development of ischemia due to severe twisting of the intestines; and iv) inflammation of the entire intestinal wall (enteritis) or the covering of the intestine (peritonitis) these proinflammatory mediators can lower the pain threshold.10 A case study evaluating low-quality hay and dietary changes in horses' risk of colic, noted that these dietary changes can lower the gastrointestinal $\mathrm{pH}$, change the production of volatile fatty acids, and change the balance of the microbiome in the hindgut, resulting in a higher risk of colic. ${ }^{11}$ By using metagenomic sequencing, veterinarians would be able to assess if the colic is due to dietary or physiological changes.

\section{Starch induced colic}

Starch constitutes the major storage of carbohydrates of grains and some plants such as C4 plants and legumes. By purposefully overloading a horse with starch, lactate production increases and hindgut $\mathrm{pH}$, fiber digestion, and volatile fatty acid (VFA) production decrease, leading to potential life-threatening gastrointestinal diseases resulting in colic.6,12 The concept of starch overloading to induce colic, is based off the first reliable starch induced method by Garner (1978). According to a previous study, colic can be induced by a starch bolus dose of $17.6 \mathrm{~g} / \mathrm{kg} /$ body weight of $85 \%$ starch and $15 \%$ wood cellulose flour, which can be prepared gruel and administered directly to the stomach via stomach tube. ${ }^{6}$ Changes to the balance of the hindgut microbiome have been noted to occur within the first 24-36 hours, post induction. ${ }^{13}$ After induction, samples can be collected from the cecum or feces and analyzed using NGS to identify bacteria present and compare to the baseline samples. These results could help identify which strains of bacteria promote a higher risk of colic. In addition, blood work, vitals, and a multitude of parameters including VFAs, lactate levels, and glucose can be collected to give a complete picture as to what is happening metabolically in a horse during colic. Even though colic is the number one killer in horses, little is known about the factors and mechanisms of the condition. There has also been limited work conducted in regards to studying the microbial structure and function of the horse's hindgut. ${ }^{14}$ This may be a result of the complexity of the microbiome in the hindgut or due to preliminary studies only culturing bacterial samples from feces or intestinal content. ${ }^{6}$ Maintenance of colonic health is essential to avoid disorders such as colic. By conducting studies that induce colic in horses by starch overloading methods, in a controlled environment, and using NGS, scientists could be able to obtain a more detailed picture of the condition and identify the shifts in bacteria populations. This information could potentially provide information on how to prevent colic, and what changes are being made to the hindgut's microbial population, which is vital information about the maintenance provided by horse caretakers and owners.

\section{Signs}

The severity of the clinical signs does not directly correlate to the severity of the condition. ${ }^{1}$ The most common sign of colic in a horse is excessive pawing or scraping at the ground. Horses may also display frequent stretching and urination, flank watching, biting at their stomach, repeated lying down and rising, rolling, groaning, excessive salivation, loss of appetite, decreased fecal output, and dark mucous membrane color.10 Horses will also have an increased heart rate due to pain and decrease circulation. However, all of these signs may not be displayed during a bout of colic. In a Colorado State University case study, they observed that in most cases of mild colic, horses displayed normal heart rate, temperature, and pulse rate. 15 In the case study, the veterinarian was able to diagnose the horse with mild colic due to lack of gut sounds. With this diagnosis, the veterinarian recommended that the horse be placed on analgesics and electrolytes. 15 However, without the cause being identified, the horse's colic became more severe and surgical intervention was needed. 15 Therefore, it is important to be able to identify a few of these signs, which indicate abdominal pain, in addition to the cause of colic. In the Colorado State University case study, if the veterinarian would have identified the cause of colic to be related to an unbalanced hindgut microbiome, they would have more than likely not have prescribed analgesics, which are known to have adverse side effects for the cecum microorganisms. ${ }^{15}$ In conclusion, if NGS could be used to properly identify the cause of colic, it could help veterinarians prescribe treatments that are more effective for the type of colic the horse may have. 10

\section{Diagnosis and treatment}

To diagnose colic in a horse, a veterinarian should give a thorough examination. The veterinarian should take into account the history and previous problems or previous bouts of colic to help identify the cause. When obtain- ing a history of a colic case, it is important to know the general types of diseases that can cause colic, including flatulent colic, strangulation obstruction, non-strangulation obstruction, enteritis, peritonitis, ulceration, and unexplained colic. ${ }^{10}$ During the physical examination, the variation in sounds observed during auscultation, over several areas of the abdomen and thorax, will help identify if the colic is caused by gas, fluid, or lack of movement. Rectal examination and ultrasonography evaluation can also add in diagnosing colic and the possible cause. Once a diagnosis has been made; colic can be treated with medication, to alleviate pain, a nasogastric tube, to alleviate bloating, or surgically, for intestinal twists. However, surgical intervention is expensive and is an extremely invasive abdominal procedure, with a mortality rate of $31-44 \%$, which requires intensive postoperative aftercare. 16 According to an article from Colorado State University's Veterinary Teaching Hospital, baseline costs of an abdominal surgery is $\$ 2800$ and upwards of $\$ 12,000$, if dead tissues and intestines need to be removed. On average, a majority of the surgeries performed to treat colic, cost between $\$ 3500-5500.1,17$ In addition to this financial burden, a case study conducted on 341 horses' survival rate longterm post-operation noted that horses experienced marked mortality ten days postsurgery. 18 Survival rate peaked at $75 \%$ at 600 days post-operation. 18 The high mortality rate from colic surgery, increase the demand for prevention of colic, in hopes to lower the rate of surgeries being performed to help colic. In addition, in the same case study, 100 horses out of 341 horses that underwent surgery, suffered from a recurrence of colic. 18 To avoid getting to the severity of surgical intervention, horse caretakers and owners need to be aware of how to prevent colic and minimize the risks of recurrence.

\section{Prevention}

Not only does identifying the cause of colic help with treatment, but it can also help with prevention. Some of the more common preventative measures include restricting simple carbohydrate consumption, including sugars from feeds containing molasses, provide clear, clean drinking water, prevent ingestion of dirt and sand by elevating the feeding surface, keep on regular feeding schedules and if changes need to be made, add them gradually, and regular maintenance including deworming and dentals. In a case study conducted on 341 colic cases noted that $4 \%$ of the horses that were diagnosed with colic, suffered from bacterial infections. ${ }^{18}$ In addition, a case study conducted with 606 horses, observed that $13 \%$ of the 
colic cases were from an unknown cause, which hindered the horses' rate of survival and treatment plan. ${ }^{19}$ A short-term case study was conducted observing survival rate of 300 horses post-surgical treatment of colic. ${ }^{20}$ The study concluded that the short-term survival rate ranges from $70.3 \%$ to $83.1 \%$, and that the cause of colic plays a major role in the percentage of survival post surgery. ${ }^{20}$ This study suggests a need for more specific diagnoses, but also the cause of colic, which could be provided with NGS. ${ }^{20}$ Overall, prevention of colic comes down to good digestive health and management.1,2

\section{Revolutionizing preventing, diagnosing, and treating colic}

Natural grazing behavior of horses is not the same as how we feed them currently. Due to drastic changes in the diet and environment, overloading the system with starches, and stress, the sensitive balance of the cecum microbiome can become unbalanced. This shift can cause proliferation of opportunistic and pathogenic bacteria, which can result in colic and other gastrointestinal conditions. While colic is still the number one cause of mortalities in the horse industry, more than $17 \%$ of cases are still perplexing veterinarians, either from bacterial infections, the hindgut microbiome being unbalance, or unknown causes. If horses had an analysis of their microbiome performed, this could help horse caretakers and owners know if they should supplement their horses with probiotics or other antimicrobial supplements, to maintain the balance of the hindgut microbiome. In addition, when horses are being diagnosed with colic, by taking a fecal sample and running NGS to identify what bacteria is present in their gastrointestinal microbiome, this could provide vital information about what the proper treatment should be. Lastly, NGS can help with treatment of colic. If a bacterial infection or an unknown cause is concluded for being the reason of colic, veterinarians can prescribe specific antibiotics or medication to target specific pathogenic bacteria in the gastrointestinal tract. Also, by conducting experiments using metagenomics, scientists can achieve a clearer picture of bacteria that are being killed and that are proliferating. This information can make diagnosing, treating, and preventing colic a more precise science, which in return can help the overall well being of horses and decrease mortality rates from colic.

\section{References}

1. USDA Animal Plant Health Inspections Service. National economic cost of equine lameness, colic, and equine protozoal myeloencephalitis (epm) in the United States. Virginia Cooperative Extension Newsletter Archive. 2001. Available from: http://www.sites.ext.vt.edu/newsletterarchive/livestock/aps-01_12/aps-0446.html

2. University of Kentucky. Colic in horses: General review. Blood-Horse. 2010. Available from: http://www. xbloodhorse. com/horse-racing/articles/55099/colic-inhorses-general-review.

3. Bentz BG. Digestion in the horse. The Horse. 2014. Available from: http://www.thehorse.com/articles/33228/di gestion-in-the-horse

4. Fraga M, Perelmuter K, Delucchi L, Zunuino P. Equine native microbiota as a source of beneficial microbes. In: Horses: Biology, Domestication, and Human Interaction. Montevideo, Uruguay: Nova Science Publisher, Inc; 2011. pp 111-20.

5. Costa MC, Arroyo LG, Allen-Vercoe E, et al. Comparison of fecal microbiota of healthy horses and horses with colitis by high throughput sequencing of the V3-V5 region of the $16 \mathrm{~s}$ rrna gene. PLoS ONE 2012;7:e41484.

6. Milinovich GJ, Klieve AV, Pollitt CC, Trott DJ. Microbial events in the hindgut during carbohydrate-induced equine laminitis. Vet Clin Equine 2010;26:79-94.

7. Illumina. Illumina sequencing technology. Illumina, Inc. 2010. Available from: http://www.illumina.com/documents/products/techspotlights/techspotlight_sequenc ing.pdf

8. Illumina. Metagenomics 2nd Edition: a review of publications featuring illumina technology. Illumina Inc. 2013. Available from: http://www.openexome. pl/Files/Application/metagenomics_resear ch_review.pdf

9. Berg EL, Fu CJ, Porter JH, Kerley MS. Fructooligosaccharide supplementation in the yearling horse: effects on fecal ph, microbial content, and volatile fatty acid concentrations. J Anim Sci 2005;83:154953.

10. Moore JN. Overview of colic in horses. Merck Veterinary Manual. 2013. Available from: http://www.merckvetmanual.com/ $\mathrm{mvm} /$ digestive_system/colic_in_horses/ov erview_of_colic_in_horses.html

11. Cohen N, Gibbs P, Woods A. Dietary and other management factors associated with equine colic. J Amer Vet Med Assoc 1999;45:96-8.

12. Hussein HS, Vogedes LA, Fernandez GCJ, Frankeny RL. Effects of cereal grain supplementation on apparent digestibility of nutrients and concentration of fermentation end-products in the feces and serum of horses consuming alfalfa cubes. J Anim Sci 2004;82:1986-96.

13. Biddle AS, Black SJ, Blanchard JL. An in vitro model of the horse gut microbiome enables identification of lactate-utilizing bacteria that differentially respond to starch induction. PLoS ONE 2013;8: e77599.

14. Medina B, Girard ID, Jacotot E, Julliand V. Effects of a preparation of saccharomyces cerevisiae on microbial profiles and fermentation patterns in the large intestine of horses fed a high fiber or a high starch diet. J Anim Sci 2002;80 2600-9.

15. Colorado State University. Case file: A challenging colic case. E-Rounds. 2013;3(6).

16. Ihler CF, Venger JL, Skjerve E. Evaluation of clinical and laboratory variables as prognostic indicators in hospitalized gastrointestinal colic horses. Acta Vet Scand 2004;45:109-18.

17. Colorado State University. Colic surgery cost estimates. Available from: http://csucvmbs.colostate.edu/Documents/equinemedicine-surgery-colic-cost-estimates.pdf.

18. Proudman CJ, Smith JE, Edwards GB, French NP. Long-term survival of equine surgical colic cases. Part 1: patterns of mortality and morbidity. Equine Vet $\mathrm{J}$ 2002;34:432-7.

19. Abutarbush M, Carmalt JL, Shoemaker RW. Causes of gastrointestinal colic in horses in western Canada: 606 cases (1992 to 2002). Can Vet J 2005;46:800-5.

20. Mair TS, Smith J. Survival and complication rates in 300 horses undergoing surgical treatment of colic. Part 1: short term survival after a single laparotomy. Equine Vet J 2005;37:296-302. 